Article

\title{
Fault Parameter Estimation Using Adaptive Fuzzy Fading Kalman Filter
}

\author{
Donggil Kim ${ }^{1}$ and Dongik Lee ${ }^{2, *}$ \\ 1 Department of Robot Engineering, Kyungil University, Gyeongsan 38428, Korea \\ 2 School of Electronics Engineering, Kyungpook National University, Daegu 41566, Korea \\ * Correspondence: dilee@knu.ac.kr; Tel.: +82-53-940-8686
}

Received: 30 May 2019; Accepted: 8 August 2019; Published: 13 August 2019

\begin{abstract}
Early detection and diagnosis of wind turbine faults is critical for applying a possible maintenance and control strategy to avoid catastrophic incidents. This paper presents a novel method to estimate the parameter of faults in a wind turbine. In this work, the estimation of fault parameters is reformulated as the state estimation problem by augmenting the parameters as an additional state. The novelty of the proposed method lies in the use of an adaptive fuzzy fading algorithm for the adaptive Kalman filter so that the convergence property during the estimation of fault parameter can be improved. The performance of the proposed method is evaluated through a set of numerical simulations with both linear and non-linear models.
\end{abstract}

Keywords: fault; fuzzy; kalman filter; parameter estimation

\section{Introduction}

System faults can be classified into three types: (a) actuator faults, (b) sensor faults, and (c) components faults. The actuator faults and sensor faults are commonly modeled as additive perturbations of the system dynamics. The component faults, which are associated with the change of the dynamics, can be modeled as a multiplicative quantity to the state variables. A general fault model for the linear system is given as follows [1]:

$$
\begin{gathered}
x(t+1)=\left(A+\Delta A_{c}\right) x(t)+\left(B+\Delta B_{c}\right) u(t)+E_{1}(t) n_{1}(t)+B f_{a}(t) \\
y(t)=\left(C+\Delta C_{c}\right) x(t)+\left(D+\Delta D_{c}\right) u(t)+E_{2}(t) n_{2}(t)+f_{s}(t)
\end{gathered}
$$

where $f_{a}(t)$ and $f_{s}(t)$ are actuator faults and sensor faults, $\Delta A_{c}, \Delta B_{c}, \Delta C_{c}$, and $\Delta D_{c}$ are component faults, $n_{1}$ and $n_{2}$ are model uncertainties, $E_{1}$ and $E_{2}$ are weighting matrices.

The time characteristics of system faults are classified into three types: (a) abrupt, (b) incipient, and (c) intermittent (Figure 1) [2]. Abrupt faults are caused by hardware damages, while incipient faults are caused by a slow parametric change, such as the effect of aging. Intermittent faults are considered as the most difficult fault category to detect because they appear and disappear repeatedly. 

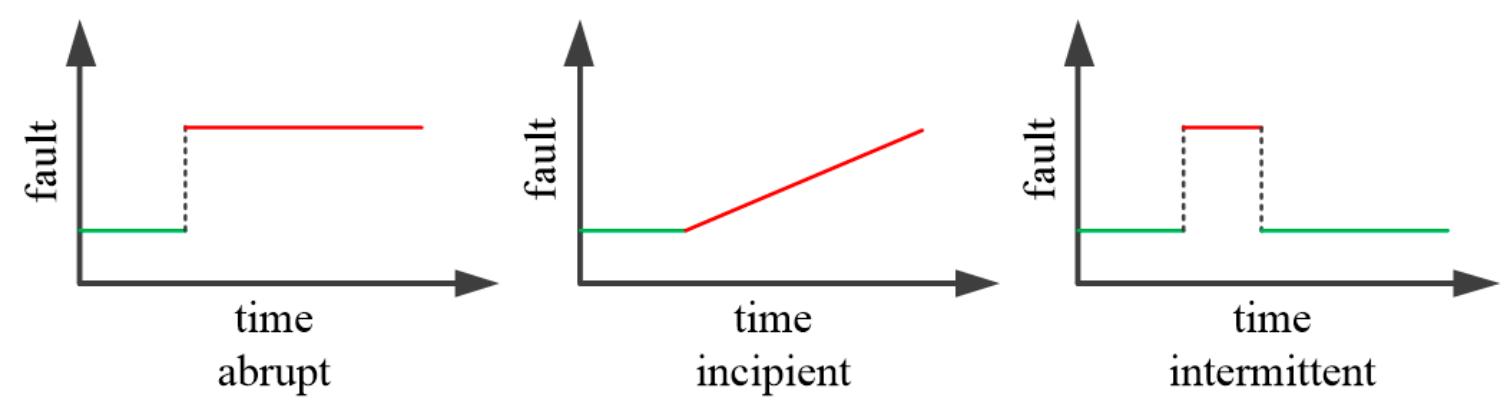

Figure 1. Fault classification according to time characteristics.

In general, fault detection and diagnosis (FDD) methods are categorized into model-based methods and data-based methods [3]. A large number of works in FDD have been developed using various approaches including observers, (joint) Kalman filters, recursive least square (RLS) algorithms, principal component analysis (PCA), expert systems, etc. PCA and expert systems are a data-based method that utilizes a large amount of historical data. The data is then transformed and presented as a priori knowledge for diagnosing the system. The approach with observers and Kalman filters is a typical model-based method that utilizes the difference (i.e., residual) between the measurement output and the estimated output. When no fault occurs, the mean of the residual will be close to zero. Conversely, faults may result in the mean of the residual deviated from zero. The approach with RLS algorithms and joint Kalman filters can be seen as a model-based method by which system parameters are directly estimated through the input-output relationship.

Meanwhile, FDD methods have to consider cooperation with fault-tolerant control (FTC). FTC will more effectively satisfy the given control objectives if FDD provides appropriate information which is needed for reconfiguration of the control structure. Typical information used in FTC includes fault parameters and fault models. For example, FTC using an adaptive control strategy requires the exact value of fault parameters [3], for which an accurate parameter estimation technique is essential.

The estimation of fault parameters can be reformulated as the state estimation problem by augmenting the parameters as additional states, which is known as the augmented Kalman filter or joint Kalman filter. The joint Kalman filter has been studied for the problem of estimating the states and the parameters simultaneously. The joint Kalman filter was first proposed in [4-6]. However, the conventional approach cannot estimate the parameters that are repeatedly changing due to system faults. As Kalman filters depend on the accuracy of a priori knowledge about the system model, any unknown information may seriously degrade the estimation performance. When a conventional joint Kalman filter is adopted to estimate the fault parameter of a dynamic system, incomplete information of the model will result in a serious bias or divergence on the estimation.

To overcome this problem, researchers have studied adaptive Kalman filter techniques [7,8], which can be classified into two types: (a) the innovation-based adaptive estimation (IAE) and (b) the multiple-model-based adaptive estimation (MMAE). In this work, IAE-based adaptive Kalman filters are examined. Many works on the IAE approach have focused on how to adapt the filter statistical information, which is the matrices $Q$ and $R$, for improving the filter performance. In contrast, a relatively small number of researchers have paid attention to the adaptation of error covariance matrix $P$, of which approach is known as the adaptive fading Kalman filter (AFKF). Note that AFKF can be significantly effective for the estimation of the fault parameters. Incomplete information about the dynamic equation caused by faults in the system can be compensated by increasing the magnitude of the error covariance matrix using a forgetting factor. Meanwhile, the convergence property during the estimation procedure becomes a critical issue when the estimation of fault parameter is combined with an FTC based on adaptive control. In AFKF, the convergence property is determined by the forgetting factor. A large value of forgetting factor leads to a sluggish estimation transition in the transient phase, while a small value of forgetting factor results in the poor estimation of the parameter change caused 
by the fault. Consequently, it is necessary to regulate the forgetting factor so as to improve both the estimation transition and its convergence time.

In order to improve the convergence property during the estimation of fault parameters, this paper presents a novel adaptive fuzzy fading Kalman filter in which the forgetting factor is determined by a fuzzy system. The use of the fuzzy system is an attractive approach for Kalman filtering, not only because of its simplicity but also its effectiveness. The benefit of a fuzzy system in Kalman filtering has been verified through various studies [9-11].

The remaining part of the paper is organized as follows. In Section 2, the existing works on the Kalman filter and joint Kalman filter are discussed. In Section 3, the proposed adaptive fuzzy fading Kalman filter is explained. A set of numerical simulations are presented in Section 4, to demonstrate the effectiveness of the proposed method, followed by concluding discussions in Section 5.

\section{Parameter Estimation with Kalman Filter}

\subsection{Kalman Filter}

Consider the following linear discrete-time stochastic system represented by

$$
\begin{aligned}
& x_{k+1}=A x_{k}+B u_{k}+w_{k} \\
& z_{k}=H x_{k}+v_{k}
\end{aligned}
$$

where $x_{k} \in R^{n}, \mathrm{z}_{k} \in{ }^{p}$ and $u_{k} \in R^{m}$ are state, measurement and input vectors, respectively. $A \in R^{n^{*} n}$ is the state transition matrix, $B \in R^{n^{*} m}$ is the input matrix and $H \in R^{p^{*} n}$ is the measurement matrix. The noise sequence $w_{k}, v_{k}$ are zero mean uncorrelated gaussian random sequences with

$$
E\left[\left[\begin{array}{c}
w_{k} \\
v_{k}
\end{array}\right]\left[\begin{array}{c}
w_{j} \\
v_{j}
\end{array}\right]^{T}\right]=\left[\begin{array}{cc}
Q_{k} & 0 \\
0 & R_{k}
\end{array}\right] \delta_{k j}
$$

where $Q_{k}>0, R_{k}>0$, and $\delta_{k j}$ is the Kronecker delta function. $E[\cdot]$ is the statistical expectation, and the superscript $T$ denotes transpose. $Q_{k}$ is the process noise covariance matrix, and $R_{k}$ is the measurement noise covariance matrix.

The Kalman filter algorithm consists of two groups of equations,

(i) Time update (or prediction) equations:

$$
\begin{aligned}
& \hat{x}_{k}^{-}=A \hat{x}_{k-1}+B u_{k-1} \\
& P_{k}^{-}=A P_{k-1} A^{T}+Q_{k-1}
\end{aligned}
$$

These equations project, from time step $k-1$ to step $k$, the current state and the error covariance estimate to obtain a priori estimates for the next time step.

(ii) Measurement update (or correction) equations:

$$
\begin{aligned}
& K_{k}=P_{k}^{-} H^{T}\left[H P_{k}^{-} H^{T}+R_{k}\right]^{-1} \\
& \hat{x}_{k}=\hat{x}_{k}^{-}+K_{k}\left[z_{k}-H x_{k}^{-}\right] \\
& P_{k}=\left[I-K_{k} H\right] P_{k}^{-}
\end{aligned}
$$

These equations incorporate a new measurement with a priori estimates to obtain an improved a posteriori estimate. Here $\hat{x}_{k}$ is an estimate of the system state vector, $K_{k}$ is commonly referred as the filter gain or Kalman gain matrix, and $P_{k}$ is the covariance matrix of estimation error, which is defined by

$$
P_{k}=E\left[\left(x_{k}-\hat{x}_{k}\right)\left(x_{k}-\hat{x}_{k}\right)^{T}\right] .
$$




\subsection{Joint Kalman Filter}

Let $\theta_{k}$ be an unknown parameter and assume that it is modeled by [12,13]:

$$
\theta_{k+1}=\theta_{k}+w_{\theta k}
$$

where, the parameter $\theta_{k}$ corresponds to a stationary process with the identity state transition matrix driven by process noise $w_{\theta k}$. To identify the unknown parameter with a Kalman filter, an augmented dynamic model is constructed with the augmented state vector defined by

$$
x_{a}=\left[\begin{array}{l}
x \\
\theta
\end{array}\right] .
$$

The augmented system yields to:

$$
\begin{aligned}
& x_{a_{k+1}}=\left[\begin{array}{c}
x_{k+1} \\
\theta_{k+1}
\end{array}\right]=\left[\begin{array}{c}
f\left(x_{k}, u_{k}, \theta_{k}\right) \\
\theta_{k}
\end{array}\right]+\left[\begin{array}{c}
w_{k} \\
w_{\theta k}
\end{array}\right] \\
& z_{k}=h\left(x_{k}, \theta_{k}\right)+v_{k}
\end{aligned}
$$

However, the augmented system is presented as a nonlinear function due to the state multiplication even if the original system in Equation (5) is linear. To handle this problem, extended Kalman filter (EKF) or unscented Kalman filter (UKF) can be used. In this paper, EKF is considered.

\subsection{Extended Kalman Filter}

The basic idea of EKF is to linearize the state space model at each time instant around the most recent state estimate, which is taken to be either $\hat{x}_{k}$ or $\hat{x}_{k}^{-}$. Once linearized model is obtained, the standard KF technique can be applied. Consider the nonlinear discrete-time stochastic system represented by

$$
\begin{gathered}
x_{k+1}=f\left(x_{k}, u_{k}\right)+w_{k} \\
z_{k}=h\left(x_{k}, \theta_{k}\right)+v_{k}
\end{gathered}
$$

Taking the linearization process with Jacobian operator given by:

$$
A_{k}=\left.\frac{\partial f}{\partial x}\right|_{x=\hat{x}_{k-1}} H_{k}=\left.\frac{\partial h}{\partial x}\right|_{x=\hat{x}_{k-1}^{-}}
$$

then, the standard Kalman filter equations can be applied.

\section{Adaptive Fuzzy Fading Kalman Filter for Fault Parameter Estimation}

The optimality of the Kalman filter can be ensured when a priori knowledge of the process/measurement noise matrices, $Q / R$, and the state transition matrix $A$ are completely known. In practical applications, however, these matrices are often insufficiently known. It is known that incomplete information makes estimations biased or even diverged $[13,14]$. To overcome this problem, various adaptive techniques have been studied. As mentioned before, component faults can be modeled as the change in the value of the dynamic system parameter. See (1). Therefore, to estimate the fault parameter via a Kalman filter, an adaptation procedure is required to handle the incompleteness of a priori information. In this paper, an adaptive fuzzy fading Kalman filter is proposed so that the convergence property during the estimation of fault parameter can be improved.

\subsection{Existing Adaptive Fading Kalman Filter}

The innovation covariance of the Kalman filter is given by

$$
C_{k}=E\left[\eta_{k} \eta_{k}^{T}\right]=H P_{k}^{-} H^{T}+R_{k}
$$


where $\eta_{k}=z_{k}-h\left(\hat{x}_{k}^{-}\right), P_{k}^{-}$and $R_{k}$ are innovations, the predicted error covariance and the measurement noise covariance, respectively. $C_{k}$ is referred to as the calculated innovation covariance $[7,8]$. The innovation covariance shows the effect of any unaccounted errors, as it is directly involved in the computations of the innovation. When the exact dynamic equation of a nonlinear stochastic system is not available, the estimation error and predicted error covariance may increase due to the effect of incomplete information. Therefore, the change of innovation covariance can be used for an adaptive filter. The increased innovation covariance is estimated by

$$
\bar{C}_{k}=\frac{1}{M-1} \sum_{i=k-M+1}^{k} \eta_{i} \eta_{i}^{T}
$$

where, $M$ is window size. $\bar{C}_{k}$ is called as the estimated innovation covariance. The relationship between $C_{k}$ and $\bar{C}_{k}$ is defined as $\bar{C}_{k}=\alpha_{k} C_{k}$. Then, the scalar value can be estimated by

$$
\alpha_{k}=\max \left\{1, \frac{1}{p} \operatorname{tr}\left(\bar{C}_{k} C_{k}^{-1}\right)\right\} \quad \text { or } \quad \alpha_{k}=\max \left\{1, \frac{\operatorname{tr}\left(\bar{C}_{k}\right)}{\operatorname{tr}\left(C_{k}\right)}\right\}
$$

where, $p$ is the dimension of $z_{k}$ and $\operatorname{tr}()$ denotes the matrix trace. The estimated innovation covariance shows the estimated filter-computed innovation covariance.

Generally, the convergence property worsened by insufficient a priori information can be improved by introducing a forgetting factor to the predicted error covariance $P_{k}$. Thus, the predicted error covariance must be increased to compensate for the effect of incomplete information on the dynamic system as follows:

$$
\bar{P}_{k}^{-}=\lambda_{k} P_{k}^{-}, \quad \lambda_{k} \geq 1
$$

where, $\lambda_{k}$ is the forgetting factor which is given by:

$$
\lambda_{k}=\frac{\operatorname{tr}\left(\alpha_{k} H P_{k}^{-} H^{T}+\left(\alpha_{k}-1\right) R_{k}\right)}{\operatorname{tr}\left(H P_{k}^{-} H^{T}\right)} .
$$

This indicates that the ratio of the innovation covariance $\alpha_{k}$ is mainly generated by $\lambda_{k}$. Therefore, $\alpha_{k}$ is almost equal to $\lambda_{k}$ (i.e., $\alpha_{k}=\lambda_{k}$ ). The predicted error covariance given as $\overline{P_{k}}=\alpha_{k} P_{k}^{-}$. This is referred to as "AFKF with rescaling-Pk."

\subsection{Design of Adaptive Fuzzy Fading Kalman Filter}

The forgetting factor of AFKF is determined as the ratio of the calculated innovation covariance and the estimated innovation covariance in the $k$-step. If any component fault occurs abruptly, it causes a step-like change in the parameter value, and then the AFKF undergoes a significant uncertainty of the state transition matrix $A_{k}$ instantaneously due to the incomplete model of Equation (5). In this case, AFKF will increase the forgetting factor to compensate for the effect of incomplete information. However, a large forgetting factor may result in the poor estimation performance, such as a longer estimation transition, in the transient phase of the filter, and eventually, the FTC based on the parameter estimation will be unstable. Therefore, it is necessary to regulate the forgetting factor to improve the estimation transition and its convergence time.

In this paper, in order to improve the convergence property of AFKF, a fading algorithm based on the fuzzy system is proposed. Since $\alpha_{k}$ is the random variable which is subject to uncertainties, it is necessary to use an approach that takes into account such uncertainties to derive relevant $\lambda_{k}$. This can be achieved with a fuzzy logic approach with rules of the kind $[15,16]$ :

$$
\text { IF < property }>\text { THEN < action }>
$$


where property and action, are the form of $\chi \in \mathrm{O}_{i}, \kappa \in \Lambda_{i}, i=1,2, \ldots$, respectively. Further, $\chi, k, \mathrm{O}_{i} / \Lambda_{i}$ are input variable, output variable, and fuzzy set, respectively.

To implement the fuzzy fading algorithm, $k$-step $\alpha_{k}$ and the change of $\alpha_{k}$ (i.e., $\Delta \alpha_{k}=\alpha_{k}-\alpha_{k-1}$ ) are used. The input variable and $\Delta \lambda_{k}$ are defined as the output of fuzzy inference system (FIS). $\Delta \lambda_{k}$ denotes the adjust term as follows:

$$
\lambda_{k f}=1+\Delta \lambda_{k}
$$

Figure 2 depicts the algorithm of the proposed adaptive fuzzy fading Kalman filter (AFFKF). The fuzzy rule for the forgetting factor is defined in Table 1. The FIS is implemented using three fuzzy sets for $\alpha_{k}$ : $\mathrm{S}=$ Small, $\mathrm{M}=$ Medium, $\mathrm{B}=\mathrm{Big}$, three fuzzy sets for the change of $\alpha_{k}$ : $\mathrm{S}=$ Small, $\mathrm{M}=$ Medium, $\mathrm{B}=\mathrm{Big}$, and four fuzzy sets for $\Delta \lambda_{k}: \mathrm{Z}=$ Zero, $\mathrm{S}=$ Small, $\mathrm{M}=$ Medium, $\mathrm{B}=$ Big. The membership functions, designed using a heuristic approach, are shown in Figure 3. The proposed AFFKF is an extension of AFKF with the fuzzy inference system that determines the appropriate forgetting factor.

Table 1. Fuzzy rules for forgetting factor of the proposed adaptive fuzzy fading Kalman filter.

\begin{tabular}{ccccc}
\hline & $\Delta \alpha_{k}$ & $\mathrm{~S}$ & $\mathbf{M}$ & $\mathrm{B}$ \\
\hline $\mathrm{S}$ & $\mathrm{S}$ & $\mathrm{Z}$ & $\mathrm{S}$ \\
$\mathrm{M}$ & $\mathrm{Z}$ & $\mathrm{S}$ & $\mathrm{M}$ \\
$\mathrm{B}$ & $\mathrm{S}$ & $\mathrm{M}$ & $\mathrm{B}$ \\
\hline
\end{tabular}

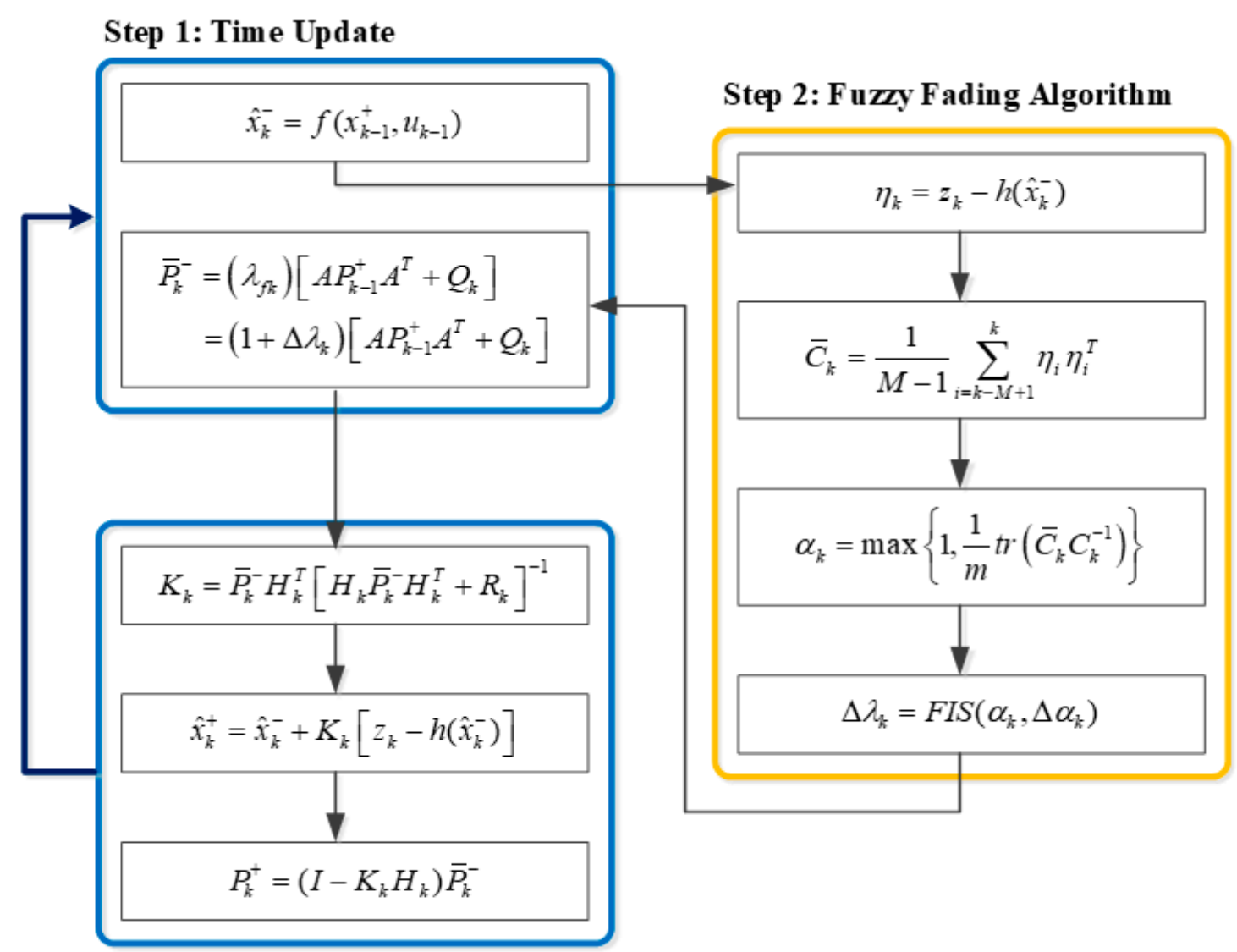

Step 3: Measurement Update

Figure 2. Computation procedure of the proposed adaptive fuzzy fading Kalman filter. 

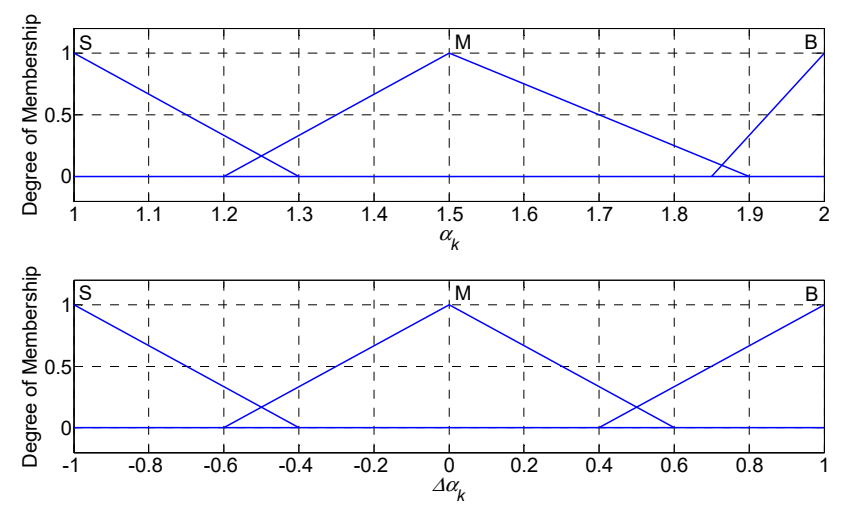

(a)

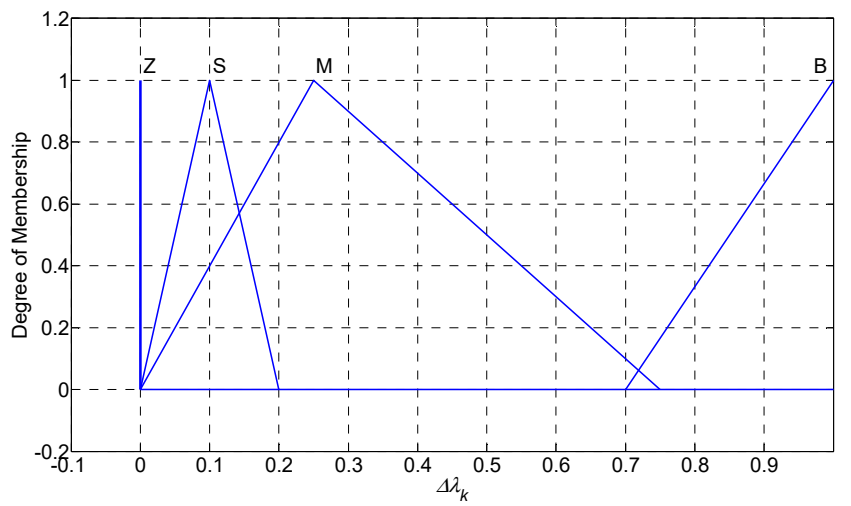

(b)

Figure 3. Membership function for adaptive fuzzy fading Kalman filter: (a) input fuzzy variables $\alpha_{k}$ and $\Delta \alpha_{k},(\mathbf{b})$ output fuzzy variable $\Delta \lambda_{k}$.

\section{Numerical Simulations}

The performance of the proposed method is evaluated with two numerical simulations: (a) the fault parameter estimation for the linear system related to a wind turbine, and (b) the fault parameter estimation for the van der pol nonlinear system.

\subsection{Fault Parameter Estimation for Linear System}

The wind turbine pitch hydraulic system model is considered as a linear system. The hydraulic pitch system is modeled as a closed-loop transfer function between the pitch angle $\beta$ and its reference $\beta_{r}$. This is a piston servo system which can be modeled by a second order transfer function as follows [17]:

$$
\frac{\beta(s)}{\beta_{r}(s)}=\frac{\omega_{n}^{2}}{s^{2}+2 \varsigma \omega_{n} s+\omega_{n}^{2}}
$$

where $\zeta$ is the damping factor and $\omega_{n}$ is the natural frequency. In normal cases, these parameters are considered as $\zeta_{0}=0.6$ and $\omega_{n 0}=11.11 \mathrm{rad} / \mathrm{s}$. The pitch system may have a fault due to the drop in hydraulic pressure, which will result in the change of system dynamics (i.e., variations of the values of $\zeta_{0}$ and $\omega_{n 0}$ ) [18]. The parameters $\zeta$ and $\omega_{n}$ in the pitch system can be reformulated as convex functions for the normal situation and the low-pressure situation as follows:

$$
\begin{aligned}
& \omega_{n}^{2}=\omega_{n 0}^{2}+\left(\omega_{n f}^{2}-\omega_{n o}^{2}\right) f \\
& \varsigma \omega_{n}=\varsigma_{0} \omega_{n 0}+\left(\varsigma_{f} \omega_{n f}-\varsigma_{0} \omega_{n 0}\right) f
\end{aligned}
$$


where $\zeta$ and $\omega_{n}$ are the current values, $\zeta_{0}$ and $\omega_{n 0}$ are the nominal values, and $\zeta_{f}$ and $\omega_{n f}$ are the values under the low pressure fault. $f$ represents the fault indicator, that is, $f=0$ denotes the normal pressure and $f=1$ denotes the low-pressure fault with $\zeta_{f}=0.9$ and $\omega_{n f}=3.42$ [19]. The process noise and the measurement noise $w_{k}$ and $v_{k}$ have the following statistics:

$$
\begin{aligned}
& E\left[w_{k}\right]=E\left[v_{k}\right]=0 \\
& E\left[w_{k} w_{j}\right]=0.01 \delta_{k-j} \\
& E\left[v_{k} v_{j}\right]=0.01 \delta_{k-j}
\end{aligned}
$$

The reference signal, $\beta_{r}$, is a sinusoidal wave with the amplitude of 2 and the frequency of $5 \mathrm{~Hz}$. The maximum value of $\alpha_{k}$ is set to 2, because a large $\alpha_{k}$ could cause a divergence. To compare the performance of each method, the following index is adopted:

$$
J(\sigma)=\sqrt{\frac{1}{n} \sum_{i=1}^{n}\left(\sigma_{i}-\hat{\sigma}_{i}\right)^{2}}
$$

where, $\sigma$ is the true parameter value, $\hat{\sigma}$ is the estimated parameter value. In this work, equation 22 is computed for the period of $5 \mathrm{~s}$ : (a) $t=0 \sim 5 \mathrm{~s}$ for the estimation of normal parameter, and (b) $t=5 \sim 10 \mathrm{~s}$ for the estimation of fault parameter.

\subsubsection{Normal Parameter Estimation}

Figure 4a shows the results of parameter estimation using the standard KF and the AFKF for the estimation of normal parameters with the initial values $\hat{\theta}_{0}=\left[\begin{array}{cc}\hat{\varsigma}_{0} & \hat{\omega}_{n 0}\end{array}\right]^{T}=\left[\begin{array}{cc}0.1 & 9\end{array}\right]^{T}$. In the simulation, to estimate the innovation covariance, the window size is $M=2$ or 6 . Both methods estimate the true values of parameters. As shown in Figure 4a, the estimation performance of AFKF depends on the window length. The filter converges to the true value quickly with a short length of the window, while the convergence rate becomes slow with a long length of the window. Figure $4 \mathrm{~b}$ shows the results of parameter estimation using the proposed AFFKF. The performance of the proposed method is compared with the existing AFKF [8]. As shown in Figure 4b, the proposed method has an advantage in the convergence rate, especially for estimating $\omega_{n}$. Table 2 shows the comparison of performance indices for four different filters: the standard KF, the AFKF with the window length of 2, the AFKF with the window length of 6, and the proposed AFFKF. From Table 2, the proposed method, AFFKF, shows the best performance in estimating the normal parameters.
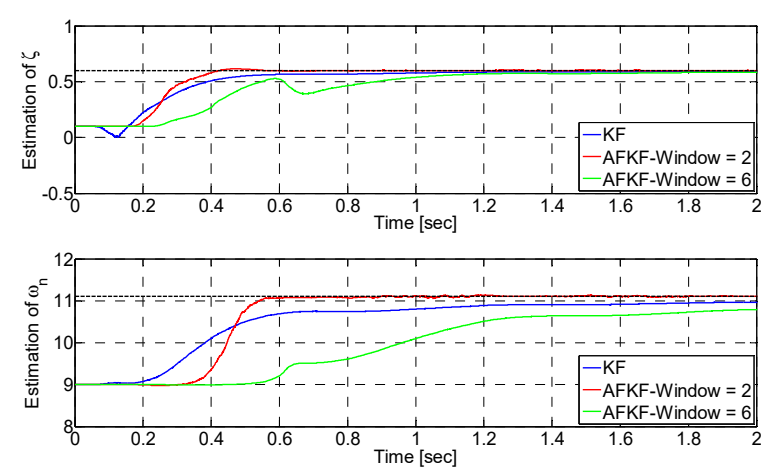

(a)

Figure 4. Cont. 

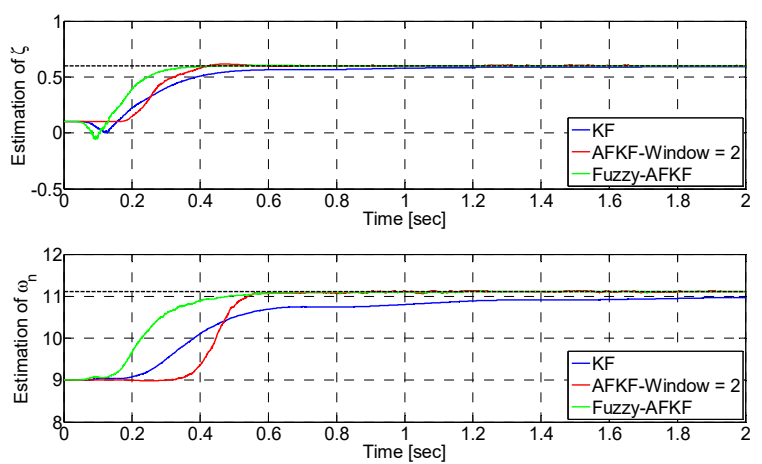

(b)

Figure 4. Results of normal parameter estimations: (a) standard Kalman filter and adaptive fading Kalman filter (AFKF), (b) the proposed AFFKF (denoted as Fuzzy-AFKF).

Table 2. Comparison of performance index for estimation of normal parameter.

\begin{tabular}{ccccc}
\hline \multirow{2}{*}{$\begin{array}{c}\text { Performance } \\
\text { Index }\end{array}$} & Standard KF & AFKF $\mathbf{~} \mathbf{M}=\mathbf{2})$ & AFKF (M = 6) & AFFKF \\
\cline { 2 - 5 } & 0.1132 & 0.1093 & 0.1444 & 0.0989 \\
$J(\zeta)$ & 0.5672 & 0.6127 & 0.8848 & 0.4250 \\
\hline$J\left(\omega_{n}\right)$ & &
\end{tabular}

\subsubsection{Fault Parameter Estimation}

In this section, a drop in the hydraulic pressure of the wind turbine pitch system is considered. Two cases of fault injection scenarios are assumed: (a) an abrupt fault case, and (b) an incipient fault case. The abrupt fault is injected at $t=5 \mathrm{~s}$, and the incipient fault starts at $t=5 \mathrm{~s}$ and ends at $\mathrm{t}=10 \mathrm{~s}$ with the continuously increasing fault indicator increases from 0 to 1 . The following figures depict estimation results for each scenario. Figure 5 a shows the estimation results for the abrupt fault scenario using the standard KF and AFKF. As shown in Figure 5a the standard KF cannot estimate the true value of the fault parameter. The estimation is biased and seems to diverge (green line). A small length of the window may induce an overestimate because the filter is sensitive to noise and uncertainties. AFKF estimates the true value of the parameter. See the results with the window size of 2 . As compared to the existing AFKF, the proposed AFFKF shows a better performance not only in the quantitative metric but also in term of the convergence property. See Figure $5 b$. Figure $5 c, d$ compare the errors in estimations with different approaches. In Figure 5d, the AFFKF presents the improved convergence property. Table 3 gives a summary of the performance index about the estimation results of the fault parameter.

Table 3. Comparison of performance index for estimation of abrupt fault parameter.

\begin{tabular}{ccccc}
\hline \multirow{2}{*}{$\begin{array}{c}\text { Performance } \\
\text { Index }\end{array}$} & Standard KF & AFKF (M = 2) & AFKF (M = 6) & AFFKF \\
\cline { 2 - 5 } & 0.4654 & 0.0741 & 0.0667 & 0.0454 \\
$J(\zeta)$ & 5.9493 & 0.7809 & 0.4712 & 0.6259 \\
\hline$J\left(\omega_{n}\right)$ & & &
\end{tabular}



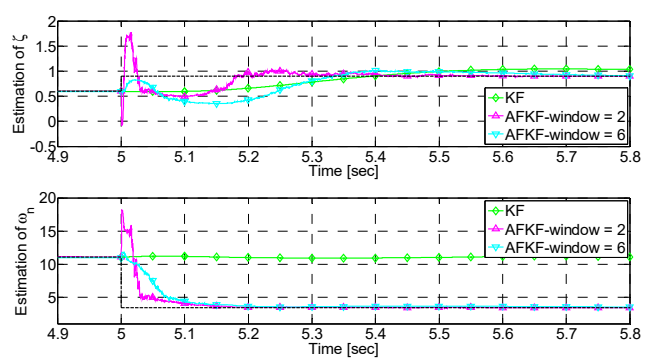

(a)

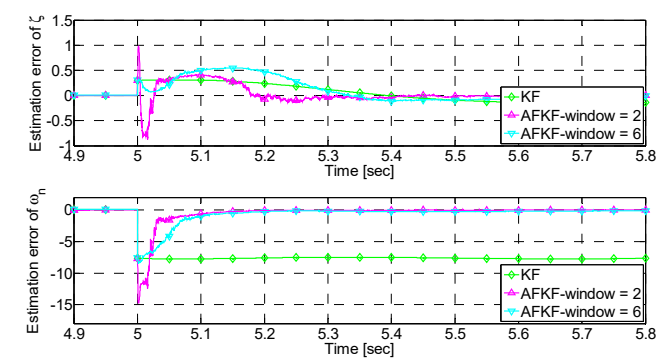

(c)
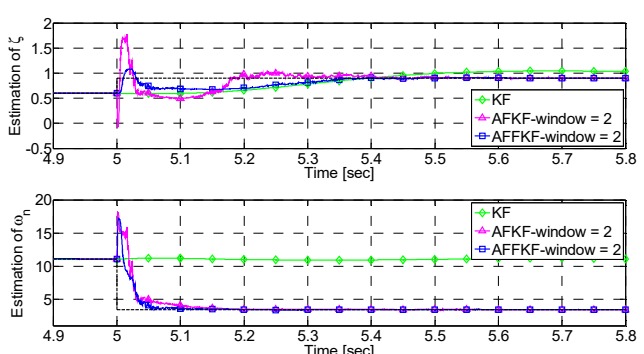

(b)

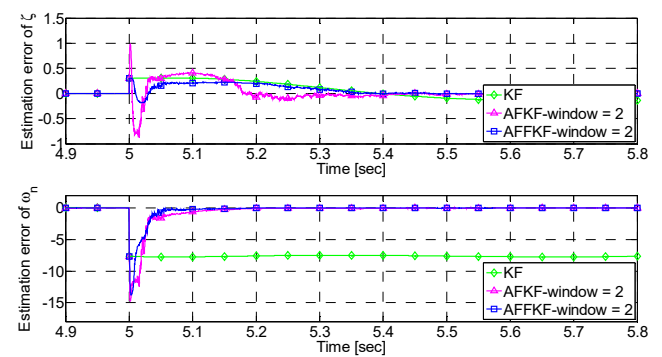

(d)

Figure 5. Results of estimation of abrupt fault parameter for linear system: (a) standard Kalman filter and AFKF, (b) the proposed AFFKF, (c) errors in estimation with standard Kalman filter and AFKF, (d) errors in estimation with the proposed AFFKF.

Figure 6 depicts the estimation results for the incipient fault scenario. The standard Kalman filter fails to estimate the true value of the parameter, and even the damping factor diverges. AFKF estimates the true value of natural frequency quickly, although an overestimate occurs around $t=10 \mathrm{~s}$. The proposed AFFKF shows the result similar to AFKF, but the performance index is slightly improved (Table 4).
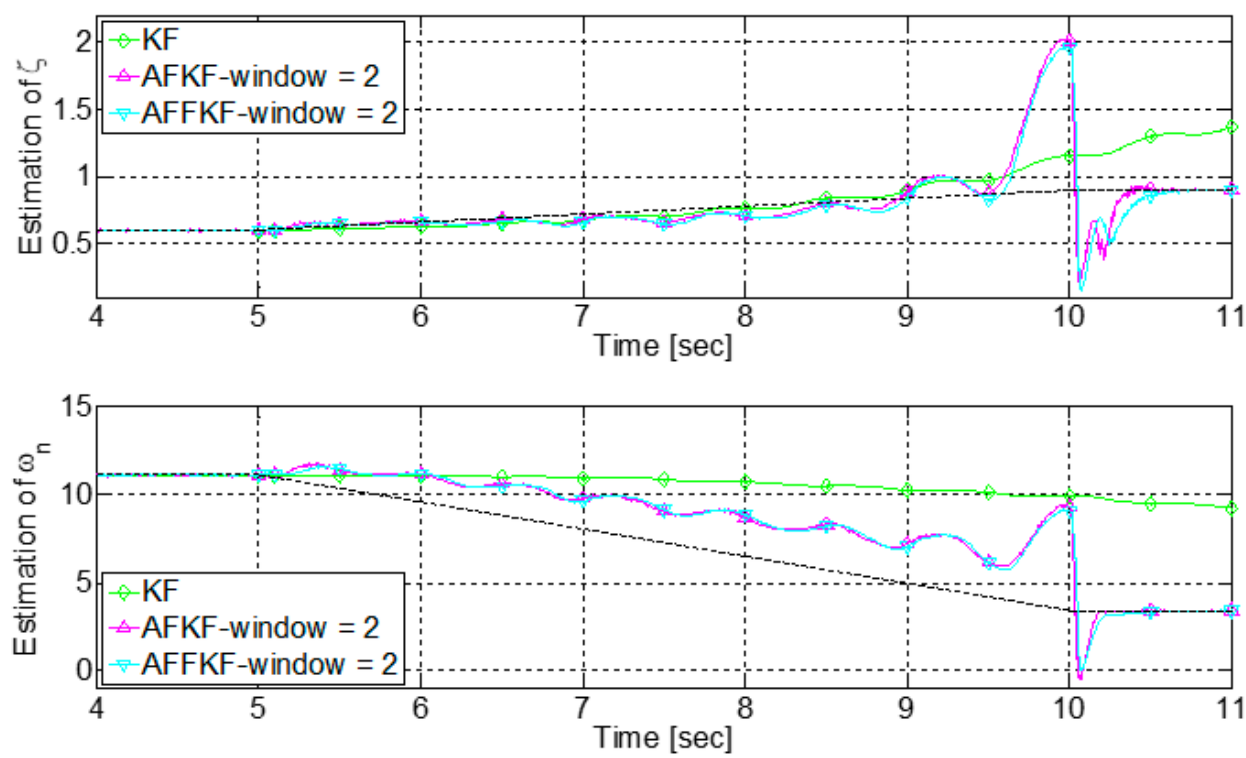

Figure 6. Results of estimation of incipient fault parameter for linear system. 
Table 4. Comparison of performance index for estimation of incipient fault parameter.

\begin{tabular}{cccc}
\hline \multirow{2}{*}{ Performance Index } & \multicolumn{3}{c}{ Approaches } \\
\cline { 2 - 4 } & Standard KF & AFKF (M = 2) & AFFKF \\
\hline$J(\zeta)$ & 0.0633 & 0.2005 & 0.1875 \\
$J\left(\omega_{n}\right)$ & 4.5716 & 1.9325 & 1.9125 \\
\hline
\end{tabular}

\subsection{Fault Parameter Estimation for Nonlinear System}

To verify the feasibility of AFFKF for a nonlinear system, the well-known van der pol model [20] is considered. The van der pol model is classically defined by a dynamic system of the following form in unforced case:

$$
\ddot{x}(t)-\mu\left[1-\frac{x^{2}(t)}{x_{0}^{2}}\right] \dot{x}(t)+\omega^{2} x(t)=0
$$

where $\mu$ is the friction coefficient, $x_{0}$ is the reference amplitude for $x$, and $w$ is the natural frequency. Equation (23) will be restricted to the case when $x_{0}=1$, as followings:

$$
\ddot{x}(t)-\mu\left[1-x^{2}(t)\right] \dot{x}(t)+\omega^{2} x(t)=0, \quad \mu, \omega>0 .
$$

The numerical simulations for parameter estimations are performed using Equation (24) with the following values:

$$
\mu_{0}=2, \omega_{0}=3, \quad \mu_{f}=0.2, \omega_{f}=2 .
$$

where, $\mu_{0}$ and $w_{0}$ denote the values of normal condition, and $\mu_{f}$ and $\omega_{f}$ denote the values of fault condition. An abrupt fault is injected at $t=50 \mathrm{~s}$. For the noisy case, the process and measurement noise $w_{k}$ and $v_{k}$ have the following statistics:

$$
\begin{aligned}
& E\left[w_{k}\right]=E\left[v_{k}\right]=0 \\
& E\left[w_{k} w_{j}\right]=0.05 \delta_{k-j} \\
& E\left[v_{k} v_{j}\right]=0.05 \delta_{k-j}
\end{aligned}
$$

The simulation results are shown in Figures 7 and 8 with initial values $\hat{\theta}_{0}=\left[\begin{array}{ll}\hat{\mu}_{0} & \hat{\omega}_{n o}^{2}\end{array}\right]^{T}=$ $\left[\begin{array}{ll}1 & 7\end{array}\right]^{T}$. The oscillatory behavior of the system is shown in Figure 7 , and the results of parameter estimations are shown in Figure 8. The conventional Kalman filter fails to estimate the true value of the fault parameter until the simulation is complete. On the other hand, both AFKF and AFFKF successfully estimate the true value of the fault parameter. However, the proposed AFFK converges much faster than AFKF does.

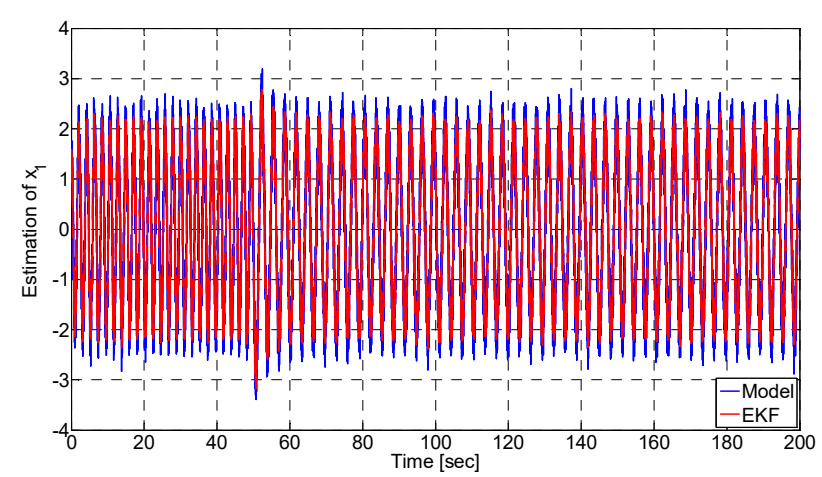

Figure 7. Estimated $x$ and measurement $y$ in van der pol model. 


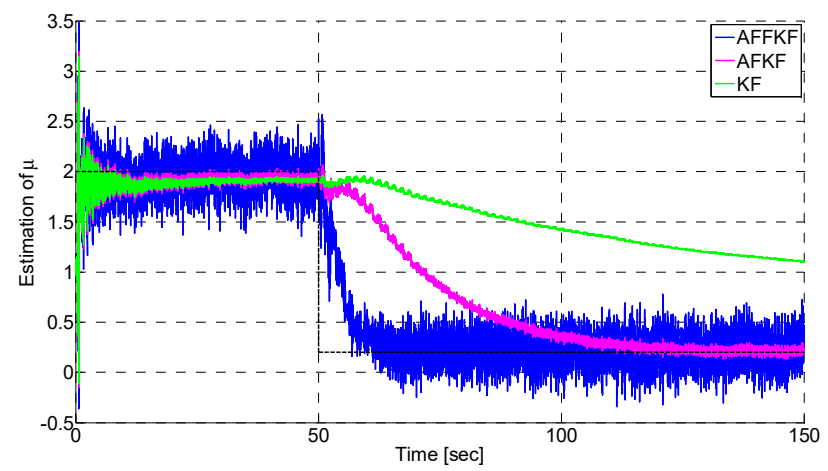

(a)

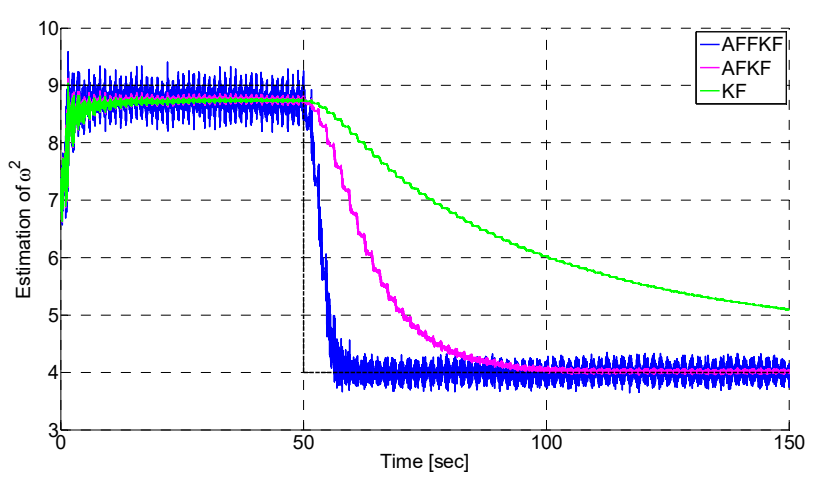

(b)

Figure 8. Results of estimation of abrupt fault parameter for van der pol model: (a) friction coefficient, (b) natural frequency.

\section{Discussion}

The estimation of the fault parameter is an attractive approach to fault detection and diagnosis (FDD), because the accurate information about the fault can be beneficial for the design of fault-tolerant control (FTC). This paper presented a parameter estimation based FDD strategy with the adaptive fuzzy fading Kalman filter (AFFKF). The proposed AFFKF can be seen as an extended version of the adaptive Kalman filter (AFKF). Using the fuzzy inference system the AFFKF is able to improve the transient phase of the filter. The effectiveness of the proposed method was evaluated through a set of numerical simulations.

Author Contributions: Conceptualization, D.K.; methodology, D.K.; software, D.K.; writing-original draft preparation, D.K.; writing-review and editing, D.L.; supervision, D.L.; project administration, D.L.; funding acquisition, D.L.

Funding: This work was supported by the National Research Foundation of Korea (NRF) grant funded by the Korea government (MSIT) (No. NRF-2017R1C1B5076020 and NRF-2017R1A2B4003008).

Conflicts of Interest: The authors declare no conflict of interest.

\section{Appendix A Effect of Large Window Length in AFKF}

The effect of a large window length is studied. In Section 4, the estimation results with the window length 2 and 6 are presented. The window length 6 shows a smoother response than the window length 2. Additional numerical study is performed for the window length 20 and 30 to verify the effect of window length. Figure A1 shows the estimation results using AFKF with the window length 2, 20 , and 30 , respectively. In contrast to the previous results, estimates with a large window length is unacceptable due to the poor transient response or divergence. Therefore, the selection of a window length is very important. 


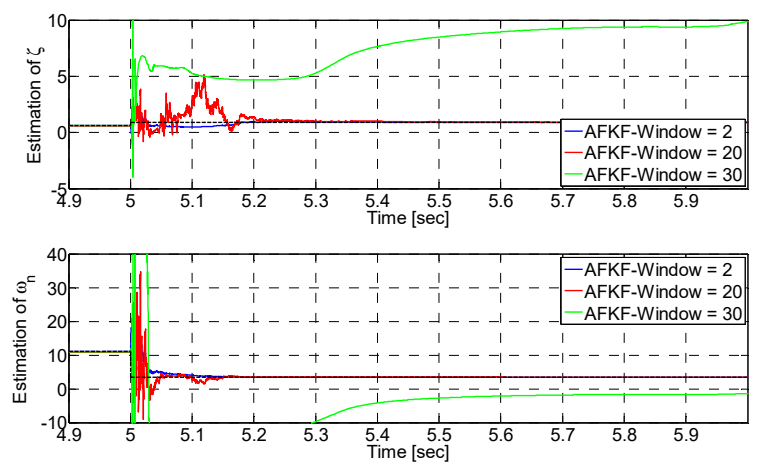

Figure A1. Results of parameter estimation using AFKF with different window lengths.

\section{References}

1. Hwang, I.; Kim, S.; Kim, Y.; Seah, C.E. Survey of fault detection, isolation, and reconfiguration methods. IEEE Trans. Control Syst. Technol. 2010, 18, 636-653. [CrossRef]

2. Edwards, C.; Lombaerts, T.; Smaili, H. A benchmark challenge. In Fault Tolerant Flight Control; Springer: Berlin/Heidelberg, Germany, 2010.

3. Zhanga, Y.; Jiang, J. Bibliographical review on reconfigurable fault-tolerant control systems. Annu. Rev. Control 2008, 32, 229-252. [CrossRef]

4. Kopp, R.; Orford, R. Linear regression applied to system identification for adaptive control systems. AIAA J. 1964, 1, 2300-2306. [CrossRef]

5. Cox, H. On the estimation of state variables and parameters for noisy dynamic systems. IEEE Trans. Autom. Control 1964, 9, 5-12. [CrossRef]

6. Nelson, L.W.; Stear, E. The simultaneous on-line estimation of parameters and states in linear systems. IEEE Trans. Autom. Control 1976, 21, 94-98. [CrossRef]

7. Kim, K. An Adaptive Filter Design for a Fault Tolerant Navigation System. Ph.D. Thesis, Seoul National University, Seoul, Korea, February 2006.

8. Kim, K.; Lee, J.; Park, C. Adaptive two-stage extended kalman filter for fault-tolerant INS-GPS loosely coupled system. IEEE Trans. Aerosp. Electron. Syst. 2009, 45, 125-137.

9. Loebis, D.; Sutton, R.; Chudley, J.; Naeem, W. Adaptive tuning of a Kalman filter via fuzzy logic for an intelligent AUV navigation system. Control Eng. Pract. 2004, 12, 1531-1539. [CrossRef]

10. Jetto, L.; Longhi, S.; Vitali, D. Localization of a wheeled mobile robot by sensor data fusion based on a fuzzy logic adapted Kalman filter. Control Eng. Pract. 1999, 7, 763-771. [CrossRef]

11. Kobayashi, K.; Cheok, K.C.; Watanabe, K.; Muneka, F. Accurate differential global positioning system via fuzzy logic Kalman filter sensor fusion technique. IEEE Trans. Ind. Electron. 1998, 45, 510-518. [CrossRef]

12. Ljung, L.; Söderström, T. Theory and Practice of Recursive Identification; MIT Press: Cambridge, MA, USA, 1983.

13. Ljung, L. Asymptotic behavior of the extended Kalman filter as a parameter estimator for linear systems. IEEE Trans. Autom. Control 1979, 24, 36-50. [CrossRef]

14. Fitzgerals, R.J. Divergence of the kalman filter. IEEE Trans. Autom. Control 1971, 16, 736-747. [CrossRef]

15. Mamdani, E.H. Application of fuzzy logic to approximate reasoning using linguistic synthesis. IEEE Trans. Comput. 1977, 26, 1182-1191. [CrossRef]

16. Takagi, T.; Sugeno, M. Fuzzy identification of systems and its applications to modeling and control. IEEE Trans. Syst. Man Cybern. 1985, 15, 116-132. [CrossRef]

17. Merritt, H.E. Hydraulic Control Systems; John Wiley \& Sons, Inc.: Hoboken, NY, USA, 1967.

18. Slotha, C.; Esbensen, T.; Stoustrup, J. Robust and fault-tolerant linear parameter-varying control of wind turbines. Mechatronics 2011, 21, 645-659. [CrossRef] 
19. Lan, J.; Patton, R.; Zhu, X. Fault tolerant wind turbine pitch control using adaptive sliding mode estimation. Renew. Energy 2018, 116, 219-231. [CrossRef]

20. Besançon, G.; Voda, A.; Jouffroy, G. A note on state and parameter estimation in a van der Pol oscillator. Automatica 2010, 46, 1735-1738. [CrossRef] 